\title{
Establishing an in vitro model of MR-T1p imaging technology to quantify nucleus pulposus tissue proteoglycans: a preliminary study
}

\author{
Zhiqiang $\mathrm{Wu}^{1 "}$, Jianqi $\mathrm{Li}^{2 \#}$, Ludan Chen ${ }^{1}$, Song Chen ${ }^{1}$, Wenquan Zhuang ${ }^{1}$ \\ ${ }^{1}$ Department of Interventional Radiology, The First Affiliated Hospital of Sun Yat-sen University, Guangzhou, China; ${ }^{2}$ Department of Radiology, \\ The First Affiliated Hospital of Sun Yat-sen University, Guangzhou, China \\ Contributions: (I) Conception and design: Z Wu, W Zhuang; (II) Administrative support: W Zhuang; (III) Provision of study materials or patients: \\ J Li; (IV) Collection and assembly of data: L Chen, S Chen; (V) Data analysis and interpretation: Z Wu, W Zhuang; (VI) Manuscript writing: All \\ authors; (VII) Final approval of manuscript: All authors. \\ "These authors contributed equally to this work. \\ Correspondence to: Wenquan Zhuang. Department of Interventional Radiology, The First Affiliated Hospital of Sun Yat-sen University, No. 58 Zhong \\ Shan Er Lu, Guangzhou 510080, China. Email: zhuangwq@mail.sysu.edu.cn.
}

Background: The aim of the present study was to construct an in vitro model of degenerated nucleus pulposus with different combinations of biochemical components, and to find an in vitro model for the early degeneration of nucleus pulposus suitable for the detection of magnetic resonance T1rho (MR-T1 $\rho$ ) sequence for the early diagnosis of degeneration of lumbar intervertebral disc.

Methods: The proteoglycan concentration gradient in the first experimental group was $5 \%$, with a concentration range of 7 samples in vitro models from 5\% to $35 \%$. The second experimental group had 15 samples with a $1 \%$ concentration gradient of proteoglycan (range, 10-24\%), with a higher water content compared with the first group. The third experimental group contained 20 samples with a concentration gradient of $1 \%$ proteoglycan (range, 10-29\%), with $75 \%$ water content. All of the in vitro models were scanned using a 3.0T GE MR. To analyze the correlation between the proteoglycan content of the in vitro model and the T1 $\rho$ value, we investigated the feasibility and stability of modeling.

Results: There was no correlation between the in vitro model proteoglycan concentration and T1 $\rho$ value in the first experimental group; however, there was a significant negative correlation between the proteoglycan concentration and T1 $\rho$ value in the second experimental group $\left(\mathrm{Y}=-3.02 \mathrm{X}+131.8, \mathrm{R}^{2}=0.852, \mathrm{P}<0.05\right)$. In the third experimental group, the proteoglycan concentration was significantly positively correlated with T1 $\rho$ value $\left(Y=3.05 \mathrm{X}+11.99, \mathrm{R}^{2}=0.834, \mathrm{P}<0.05\right)$. The comparison of the $\mathrm{T} 1 \rho$ values in the third experimental group before and 3 months after yielded an intraclass correlation coefficient value of 0.980 , indicating that the biochemical components in the third experimental group were still stable after 3 months of storage. The slope of the regression equation between the Pfirrmann grading and T1 $\rho$ value in the third experimental group was not statistically different from the volunteer group $(\mathrm{F}=0.54, \mathrm{P}=0.814)$, suggesting that the lumbar disc nucleus pulposus tissue of in vitro model samples fitted well with the volunteer group.

Conclusions: In this experiment, we successfully constructed an in vitro model of nucleus pulposus tissue proteoglycan that can be used for the quantitative evaluation of the MR-T1 $\rho$ imaging.

Keywords: Lumbar disc degeneration; nucleus pulposus tissue; proteoglycan; in vitro model; magnetic resonance T1rho

Submitted Jul 30, 2021. Accepted for publication Oct 02, 2021.

doi: 10.21037/atm-21-4297

View this article at: https://dx.doi.org/10.21037/atm-21-4297 


\section{Introduction}

Low back pain is common, and in severe cases, can affects people's quality of life and ability to work (1). Worsening lumbar disc degeneration can cause lumbar disc herniation. Therefore, it important to detect and treat degeneration of the lumbar intervertebral disc as early as possible in the clinical setting to delay or even reverse the degeneration of the intervertebral disc and prevent further deterioration.

The accurate diagnosis of early degeneration of the lumbar intervertebral disc has important clinical value and significance. Previous studies have confirmed that the early degeneration of lumbar intervertebral disc is first reflected in the content change of proteoglycans in the nucleus pulposus tissue (2); however, the non-invasive imaging methods currently used cannot quantitatively detect proteoglycans. Due to the tissue structure of the lumbar intervertebral disc, the early diagnosis of degeneration cannot be confirmed by needle biopsy. Magnetic resonance imaging (MRI) is one of the most commonly used imaging techniques for diagnosing soft tissue diseases. Many scholars have reported that magnetic resonance T1rho (MR-T1 $\rho$ ) imaging technology can quantify the changes of proteoglycan content in the nucleus pulposus tissue of early lumbar disc degeneration $(3,4)$, and that it is more sensitive than conventional magnetic resonance $\mathrm{T} 2$ weighted image (MR-T2WI) imaging and T2-mapping imaging. In addition, some scholars have found that when studying degeneration of the lumbar intervertebral disc in humans and cadavers, MR-T1 $\rho$ imaging is more sensitive than MRT2WI imaging in the early degeneration of the lumbar intervertebral disc (5). The proteoglycan concentration in the lumbar intervertebral disc nucleus pulposus tissue has been analyzed in many studies, which have found that the proteoglycan concentration has a good and positive correlation with the $\mathrm{T} 1 \rho$ relaxation time value (6-8).

However, when reviewing studies using MR-T1 $\rho$ imaging to quantitatively assess early lumbar disc degeneration, we found that in the lumbar disc nucleus pulposus tissue with the same Pfirrmann degeneration grade, significant differences in the MR-T1 $\rho$ relaxation time value have been reported (9). When we studied the correlation between the proteoglycan content in the nucleus pulposus tissue of the natural degeneration and the MRT1 $\rho$ value of the rhesus monkeys in the early stage, we found significant differences in the MR-T1 $\rho$ value when the same lumbar disc nucleus pulposus tissue was detected on different magnetic resonance equipment. Therefore, if the MR-T1 $\rho$ value measured by the magnetic resonance equipment is directly used to quantitatively evaluate the proteoglycan content of the human lumbar intervertebral disc nucleus pulposus, the MR-T1 $\rho$ value data obtained by different examination equipment of intervertebral discs with similar degrees of degeneration will also have differences, which cannot meet the requirements of a clinically accurate diagnosis of early degeneration of lumbar intervertebral disc. Therefore, when using MR-T1 $\rho$ imaging technology to quantitatively evaluate the degeneration of the lumbar intervertebral disc, an in vitro calibration model is required on clinical application to solve the detection error of the magnetic resonance T1rho (MR-T1 $\rho$ ) sequence on different machines. In the present study, we constructed a series of in vitro models of nucleus pulposus tissue with different proteoglycan concentrations to measure the MRT1 $\rho$ relaxation time of the in vitro models. We determined if there were any similar characteristics between the in vitro nucleus pulposus tissue models and the human degenerated lumbar disc nucleus pulposus tissue, providing theoretical data for the establishment of a standardized mathematical model of degenerated lumbar intervertebral disc nucleus pulposus tissue and MR-T1 $\rho$ relaxation time value in the future. We present the following article in accordance with the MDAR reporting checklist (available at https://dx.doi. org/10.21037/atm-21-4297).

\section{Methods}

\section{Proteoglycan concentration in the in vitro model of nucleus pulposus tissue}

The $75 \%$ proportion was selected as the water content of the in vitro model, which was $2.25 \mathrm{~g}$. The concentration of proteoglycan and the content of type II collagen fibers were set. Three groups were involved in the present study. In the first group, the water content and type II collagen fibers (West Asia Reagent Company, Chengdu, China) were set to 2.25 and $0.3 \mathrm{~g}$, respectively. The concentration range of proteoglycan (West Asia Reagent Company) was set to $5-35 \%$, with a concentration gradient of $5 \%$. The 2 control groups (water with proteoglycan and water with type II collagen) and all of the in vitro models in this group were not sterilized after construction. To ensure uniformity in the in vitro model, the water content in the second group of the in vitro models was increased from 2.25 to $3 \mathrm{~g}$, the content of type II collagen fiber remained $0.3 \mathrm{~g}$, and the proteoglycan concentration range was set to $10-24 \%$, 
with a concentration gradient of $1 \%$. After the model was constructed, it needed to be sterilized by ultraviolet light and sealed and stored at a low temperature $\left(10-15^{\circ} \mathrm{C}\right)$. The water content and type II collagen fibers in the third group of in vitro models was the same as that in the first group; the proteoglycan concentration range was set to $10-29 \%$, with a concentration gradient of $1 \%$. After the in vitro model in the third experimental group was constructed, it also needed to be sterilized by ultraviolet light and sealed and stored at a low temperature $\left(10-15^{\circ} \mathrm{C}\right)$.

\section{Methods of constructing an in vitro model of nucleus pulposus tissue}

First, the reagent bottle was rinsed with deionized water and then sterilized and dried. The required proteoglycan and type II collagen were weighed in a sterile laboratory, and the materials were put into the reagent bottle. The required amount of water was added using a micropipette. The materials were stirred with a magnetic stirrer (Dalong Xingchuang, Beijing, China), by ultrasonic mixing, heating and shaking to mix the molding material into a gel-like material, and finally sterilized by ultraviolet light and stored at a low temperature. The model samples in the first group were modeled with a magnetic stirrer for $30 \mathrm{~min}$, and the samples were not sterilized by ultraviolet rays or stored at low temperatures after modeling. The model samples in the second group were modeled by ultrasonic stirring for $30 \mathrm{~min}$. After modeling, the samples were sterilized by ultraviolet light and stored at a low temperature $\left(10-15^{\circ} \mathrm{C}\right)$. The model samples in the third group were heated and shaken for $24 \mathrm{~h}$ (temperature: $60^{\circ} \mathrm{C}$; the temperature was automatically increased from room temperature to $60{ }^{\circ} \mathrm{C}$ within $24 \mathrm{~h}$ ), and then ultrasonically stirred for $30 \mathrm{~min}$ for modeling. After modeling, the samples were sterilized by ultraviolet light and stored at a low temperature $\left(10-15^{\circ} \mathrm{C}\right)$. When the in vitro model failed to form a gel-like substance or there was obvious stratification, the in vitro model construction was considered to have failed.

\section{Acquisition of magnetic resonance image}

The clinical 3.0T GE magnetic resonance scanner (Discovery MR 750w 3.0T; GE Medical System, Boston, Massachusetts, USA) was used to scan the T1 $\rho$ sequence of the in vitro model. Two researchers who did not participate in the modeling selected the region of interest (ROI). Finally, the post-processing software provided by GE was employed to calculate the $T 1 \rho$ relaxation time value. The human lumbar intervertebral disc was also scanned by T1 $\rho$ sequence with the same parameter, and its $\mathrm{T} 1 \rho$ relaxation time value was measured. The human lumbar intervertebral disc was scanned using the conventional T2WI sequence and then graded by a professional according to the Pfirrmann classification.

The main parameters of the MR-T1 $\rho$ imaging technology included pulse sequence, fast spin echo (10, 30, and $60 \mathrm{~ms}$ ), repetition time: $80 \mathrm{~ms}$, echo time: $8 \mathrm{~ms}$, field of view: $160 \mathrm{~mm} \times 100 \mathrm{~mm}$, frequency (pixel bandwidth): $500 \mathrm{~Hz}$, voxel size: $0.59 \mathrm{~mm} \times 1.17 \mathrm{~mm}$, Slice thickness: $3 \mathrm{~mm}$, interslice gap: $0 \mathrm{~mm}$, number of slices: $5-7$, and acquisition time (min): $4 \mathrm{~min}$. According to the volume of the in vitro model samples, the MR-T1 $\rho$ sequence scan in the first group was scanned by small size flexible-joint phased array coils with 8-channel. The second and third groups were scanned with small size flexible-joint phased array coils with 16-channel.

\section{Image post-processing}

A double-blind experimental method was adopted to obtain the ROI. Drawing of the ROI area was done by 2 researchers. The basic conditions of selecting the ROI area were as follows (Figure 1A,1B): (I) the participator should choose at least 2 levels closing to the center. Edge area and defect part should be avoided to make the value of each region have a uniform representation; (II) the shape of the ROI was generally elliptical, and each ROI drawn was similar in size, and (III) the T1 $\rho$ relaxation time value of the ROI was measured and recorded 3 times for each sample in the in vitro model, and the average value was taken as the final statistical analysis data. The T1 $\rho$ value was calculated using a previously reported method (10). After being stored at a low temperature of $10-15{ }^{\circ} \mathrm{C}$ for 3 months, the third group of in vitro model samples was rescanned by MR-T1 $\rho$ with the same scanning parameters.

\section{Statistical analysis}

All statistical data were analyzed using SPSS version 25.0 (SPSS Inc. Chicago, IL, USA). Measurement data were expressed as mean \pm standard deviation. To test whether the T1 $\rho$ relaxation time value of the in vitro model in line with normal distribution, the Pearson correlation coefficient was used to analyze the correlation between the in vitro model proteoglycan concentration and the T1 $\rho$ value. The 

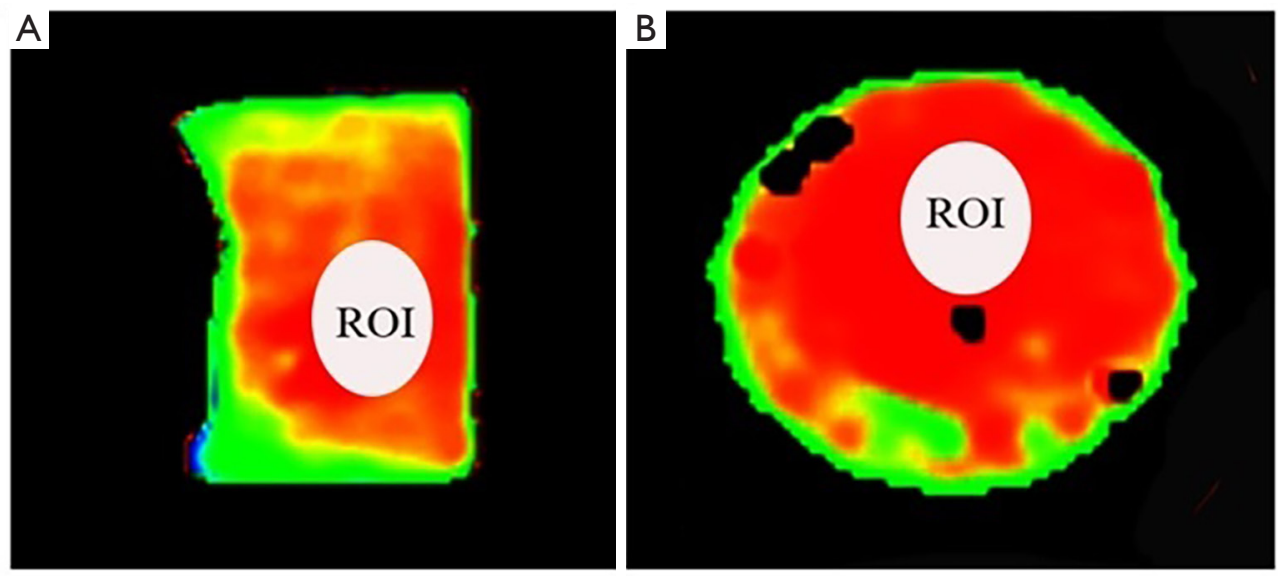

Figure 1 Schematic diagram of measurement of the in vitro model magnetic resonance T1rho (MR-T1 $\rho$ ) relaxation time value. (A) Schematic diagram of the region of interest (ROI) of the coronal position of the in vitro model. (B) Schematic diagram of the horizontal position of the ROI of the in vitro model.

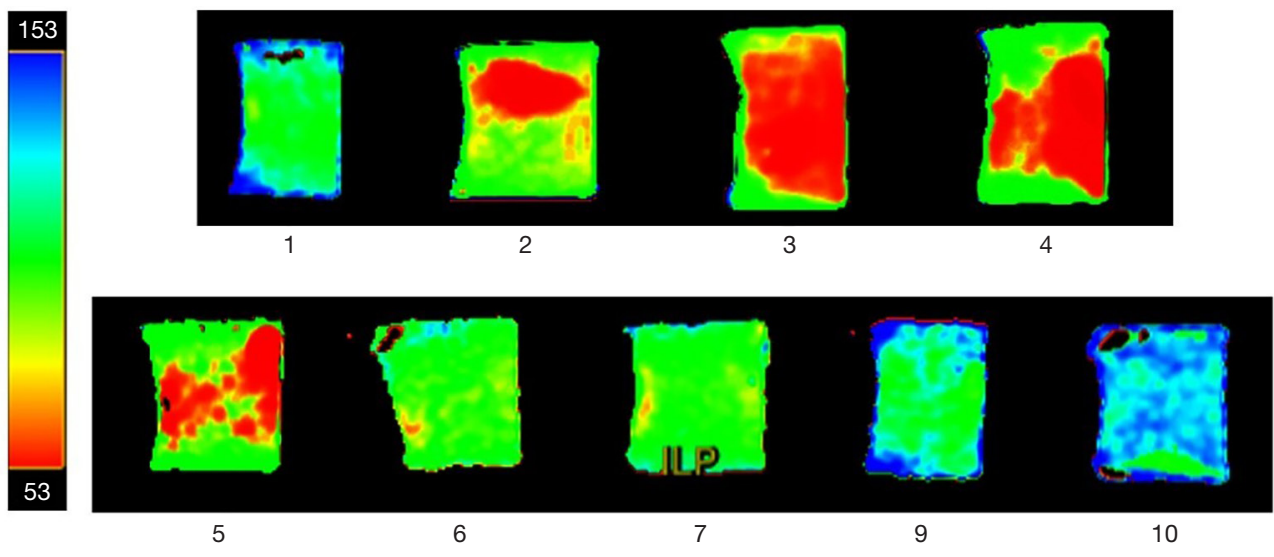

Figure 2 Magnetic resonance T1rho (MR-T1C) pseudo-color image of in vitro model samples in the first experimental group in the coronal position. There were defective areas in small amount of the samples, as indicated by the uneven color distribution.

slope of the regression equation between the T1 $\rho$ value and Pfirrmann grading of the in vitro model and volunteer lumbar disc nucleus pulposus tissue was analyzed by covariance analysis. The consistency test and evaluation of the T1 $\rho$ value of the same batch of in vitro model samples at different detection times were analyzed by intraclass correlation coefficient (ICC). $\mathrm{P}<0.05$ indicated statistical difference.

\section{Results}

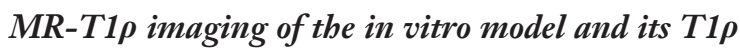 relaxation time value}

Of the 10 in vitro model samples in the first experimental group, sample 8 failed to form, so the success rate of the model making was $90 \%$. The MR-T1 $\rho$ images of the in vitro model are shown in Figure 2. The biochemical compositions of each sample successfully modeled and their corresponding T1 $\rho$ values are shown in Table 1. Of these, in vitro model group 1 was used as the control group. Type II collagen was not added to this group, and its T1 $\rho$ value was 200 ms. Proteoglycan was not added to control group 10 , with its T1 $\rho$ value was $306.1 \mathrm{~ms}$. T1 $\rho$ values of in vitro models 2-9 in the experimental group deviated significantly from the control group.

Of the 15 in vitro model samples in the second experimental group, the success rate of model making was 
Table 1 Biochemical components of the 9 model samples in the first experimental group and their T1rho (T1 $\rho)$ relaxation time values

\begin{tabular}{lccccc}
\hline Serial number & Proteoglycan $(\mathrm{g})$ & Type II collagen $(\mathrm{g})$ & Water $(\mathrm{g})$ & Proteoglycan concentration $(\%)$ & T1 $\rho$ value $(\mathrm{ms})$ \\
\hline 1 & 0.25 & 0 & 2.25 & 10 & 5 \\
2 & 0.13 & 0.3 & 2.25 & 10 & $34.3 \pm 2.0$ \\
3 & 0.28 & 0.3 & 2.25 & 15 & $75.6 \pm 4.6$ \\
4 & 0.45 & 0.3 & 2.25 & 20 & $45.0 \pm 2.2$ \\
5 & 0.63 & 0.3 & 2.25 & 25 & $54.3 \pm 2.2$ \\
6 & 0.85 & 0.3 & 2.25 & 30 & $73.4 \pm 4.0$ \\
7 & 1.10 & 0.3 & 2.25 & 35 & $63.9 \pm 4.1$ \\
10 & 1.37 & 0.3 & 2.25 & 0 & $302.2 \pm 2.1$ \\
\hline
\end{tabular}

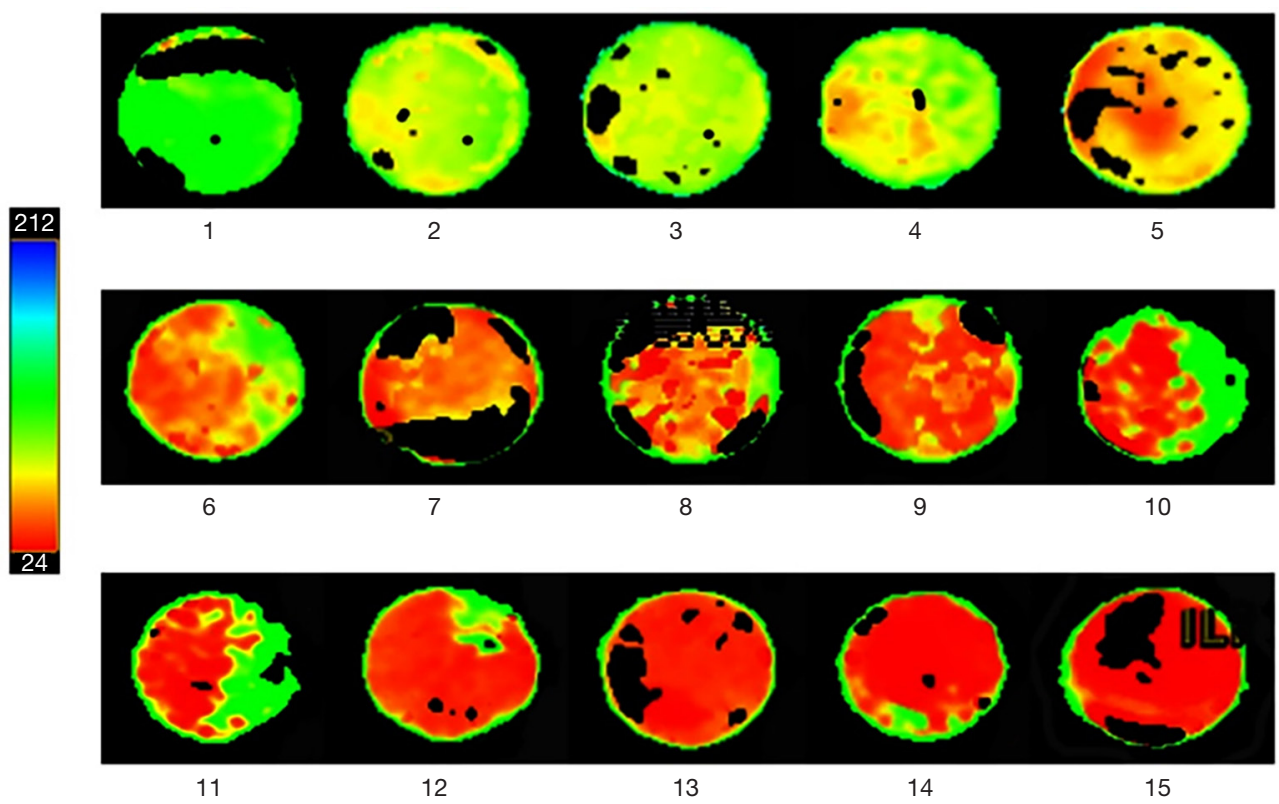

Figure 3 Magnetic resonance T1rho (MR-T1 $\rho$ ) sequence pseudo-color image of in vitro model samples in the second experimental group in the horizontal position.

$100 \%$. The MR-T1 $\rho$ images of the in vitro model sample are shown in Figure 3, and small, spot-like or patchy defects could be seen in the model sample. The biochemical compositions of each sample and their corresponding T1 $\rho$ values are shown in Table 2 .

Of the 20 in vitro model samples in the third experimental group, 5 samples were not modeled, so the success rate of the model-making process was $75 \%$. The samples modeled successfully were tested twice. The MRT1 $\rho$ images of the model samples tested for the first time are shown in Figure 4. No defect area was seen inside the model. The model samples tested for the second time were stored at $10-15{ }^{\circ} \mathrm{C}$ for 3 months, and then MR-T1 $\rho$ imaging was re-performed, with MR-T1 $\rho$ images shown in Figure 5. Of these, 4 model samples had small patchy defects inside. The biochemical components and their $\mathrm{T} 1 \rho$ values of 15 successful in vitro model samples tested twice are shown in Table 3.

The ICC was further used to test the consistency of the 2 T1 $\rho$ values before and after the cold storing of each sample. 
Table 2 Biochemical components of the model samples in the second experimental group and their T1rho (T1 $\rho)$ relaxation time values

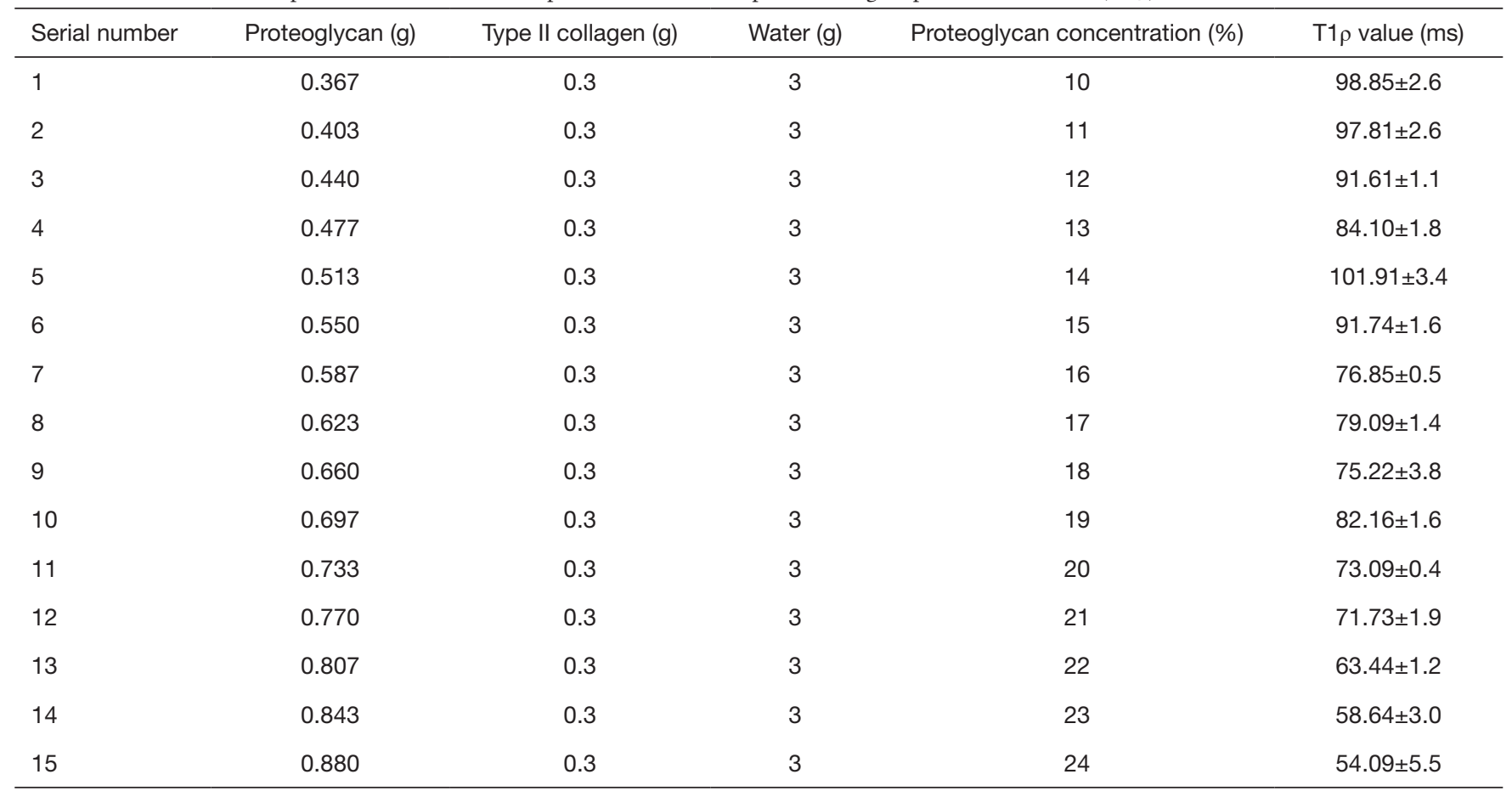

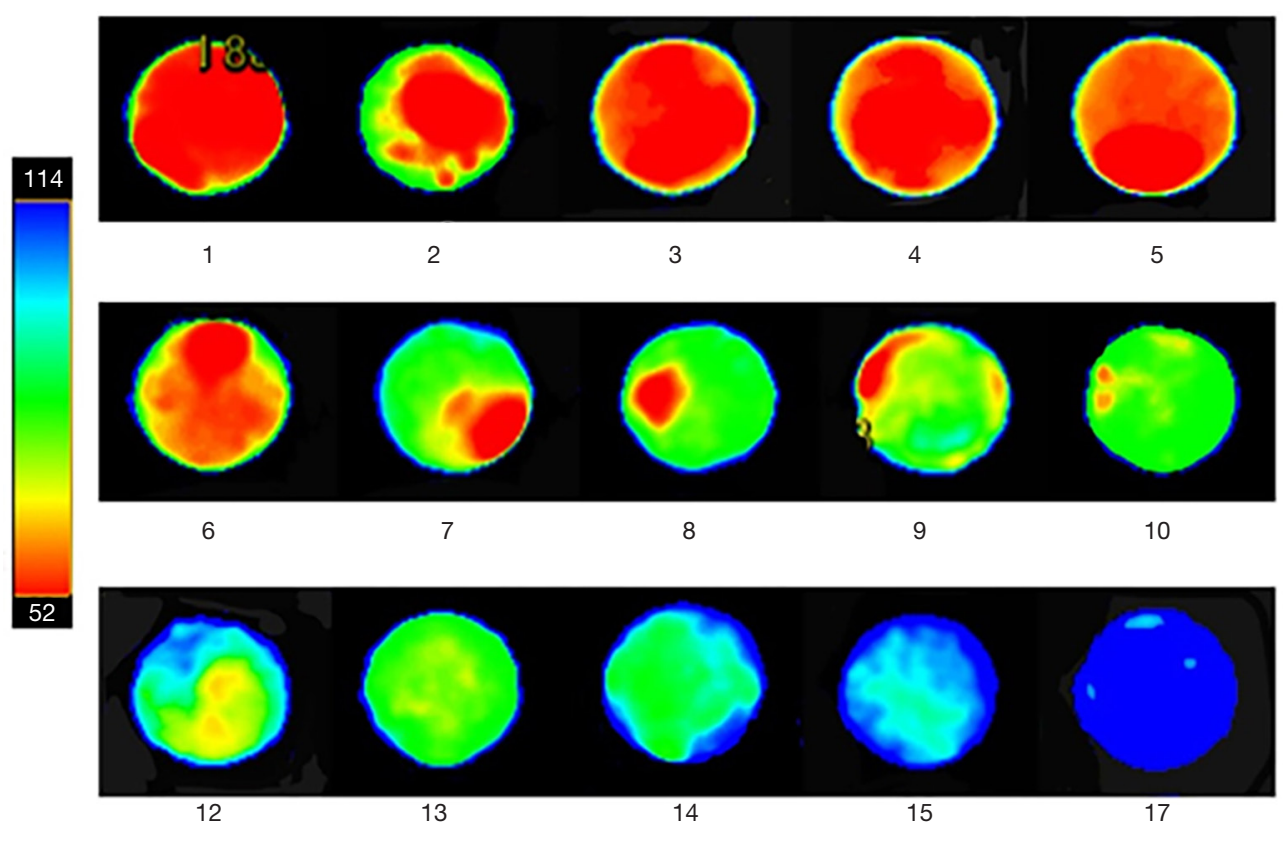

Figure 4 Magnetic resonance T1rho (MR-T1 $\rho)$ sequence pseudo-color image of in vitro model samples in the third experimental group in the horizontal position. 


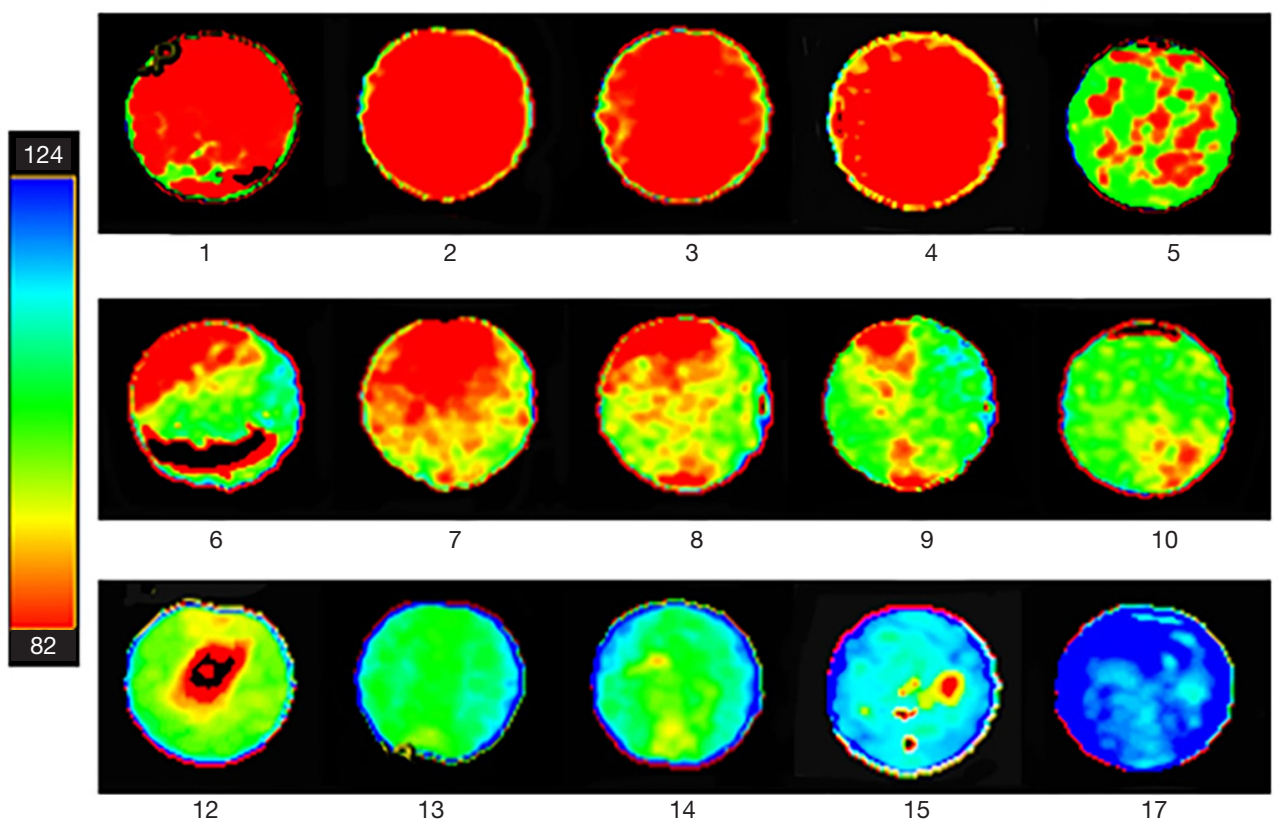

Figure 5 Magnetic resonance T1rho (MR-T1 $\rho)$ sequence pseudo-color image after 3 months modeling of the in vitro model samples in the third experimental group in the horizontal position.

Table 3 Biochemical components of the 15 sample models in the third experimental group and their 2 T1rho (T1 $\rho$ ) relaxation time values

\begin{tabular}{|c|c|c|c|c|c|c|}
\hline Serial number & $\begin{array}{l}\text { Proteoglycan } \\
\text { (g) }\end{array}$ & $\begin{array}{l}\text { Type II collagen } \\
\text { (g) }\end{array}$ & $\begin{array}{l}\text { Water } \\
\text { (g) }\end{array}$ & $\begin{array}{c}\text { Proteoglycan } \\
\text { concentration (\%) }\end{array}$ & $\begin{array}{c}\mathrm{T} 1 \rho \text { values of the first } \\
\text { test }(\mathrm{ms})\end{array}$ & $\begin{array}{l}\text { T1 } \rho \text { values of the } \\
\text { second test (ms) }\end{array}$ \\
\hline 1 & 0.283 & 0.3 & 2.25 & 10 & $44.81 \pm 2.6$ & $51.90 \pm 3.2$ \\
\hline 3 & 0.348 & 0.3 & 2.25 & 12 & $51.07 \pm 1.5$ & $51.70 \pm 2.2$ \\
\hline 4 & 0.380 & 0.3 & 2.25 & 13 & $51.63 \pm 3.4$ & $52.65 \pm 2.7$ \\
\hline 6 & 0.449 & 0.3 & 2.25 & 15 & $58.71 \pm 1.9$ & $61.23 \pm 1.3$ \\
\hline 7 & 0.486 & 0.3 & 2.25 & 16 & $63.90 \pm 1.3$ & $69.95 \pm 2.8$ \\
\hline 8 & 0.523 & 0.3 & 2.25 & 17 & $61.65 \pm 1.6$ & $63.33 \pm 2.3$ \\
\hline 13 & 0.678 & 0.3 & 2.25 & 22 & $61.46 \pm 2.3$ & $73.69 \pm 2.1$ \\
\hline 14 & 0.719 & 0.3 & 2.25 & 23 & $83.60 \pm 3.2$ & $84.36 \pm 3.3$ \\
\hline 16 & 0.761 & 0.3 & 2.25 & 25 & $92.83 \pm 0.3$ & $93.37 \pm 0.8$ \\
\hline 17 & 0.805 & 0.3 & 2.25 & 26 & $105.5 \pm 6.3$ & $109.60 \pm 7.9$ \\
\hline
\end{tabular}


A

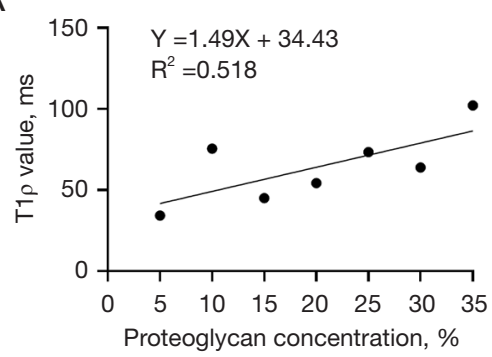

B

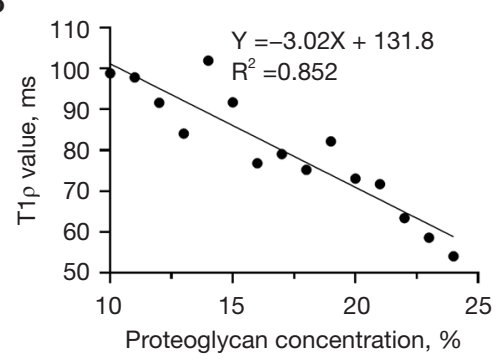

C

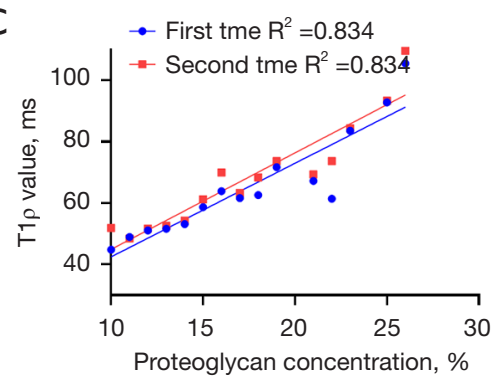

Figure 6 Correlation analysis between the proteoglycan concentration and T1rho (T1p) values of the model samples in the experimental groups. (A) Scatterplot and regression line of the proteoglycan concentration of the in vitro model samples in the first experimental group and their T1 $\rho$ values; (B) scatterplot and regression line of the proteoglycan concentration of the model samples in the second experimental group and their T1 $\rho$ values; (C) scatterplot and the regression line of the proteoglycan concentration and T1 $\rho$ values for the first and second measurements of the model samples in the third experimental group.

The results showed that the ICC of the T1 $\rho$ values of the 2 scans was 0.980 , which was greater than 0.75 , suggesting that the biochemical components of the in vitro model samples in the third experimental group were still relatively stable after being stored for 3 months.

\section{Correlation between in vitro model proteoglycan concentration and T1 $1 \rho$ value of the 3 groups}

Plotting was done by Pearson linear regression analysis, and the T1 $\rho$ relaxation time values of samples 2-9 in the first group were found to be normally distributed. The results showed that there was no correlation between the in vitro model proteoglycan concentration and $\mathrm{T} 1 \rho$ values $\left(\mathrm{Y}=1.49 \mathrm{X}+34.43, \mathrm{R}^{2}=0.518, \mathrm{P}>0.05\right)$ (Figure 6A). The T1 $\rho$ values of the 15 samples in the second group were normally distributed, as plotted by Pearson linear regression analysis. The results showed that there was a distinctly negative correlation between the proteoglycan concentration of the in vitro model samples and their $\mathrm{T} 1 \rho$ values $\left(\mathrm{Y}=-3.02 \mathrm{X}+131.8, \mathrm{R}^{2}=0.852, \mathrm{P}<0.05\right)$ (Figure 6B).

The T1 $\rho$ values of the 15 in vitro model samples in the third group were normally distributed, as plotted by Pearson linear regression analysis. The results showed that there was a distinctly positive correlation between the proteoglycan concentration in the first in vitro model samples and the T1 $\rho$ values $\left(\mathrm{Y}=3.05 \mathrm{X}+11.99, \mathrm{R}^{2}=0.834, \mathrm{P}<0.05\right)$ (Figure $6 C$ ). The comparison of the slope of the regression equation between the proteoglycan concentration in the third experimental group in the in vitro model sample and the first measurement, as well as the second measurement, showed that there was no significant difference between them $(\mathrm{F}=1.27, \mathrm{P}=0.270)$, with a good degree of fit. This finding indicated that the biochemical components of the model samples were still relatively stable after being sealed and cryopreserved.

\section{MR-T1p correlation analysis of the third experimental group in the in vitro model and lumbar intervertebral disc of the volunteers}

The MR-T1 $\rho$ imaging of the in vitro model samples were compared with the human lumbar intervertebral discs of volunteers. In this experiment, the MR-T1 $\rho$ sequence with the same parameters was used to scan the lumbar intervertebral discs of 4 adult volunteers with no low back pain, and the T1 $\rho$ values of the nucleus pulposus tissue of their intervertebral discs were measured (Table 4). T2WI sequence scanning was applied to obtain T2WI images and Pfirrmann grading evaluation to establish the regression equation between the Pfirrmann grading of the lumbar disc nucleus pulposus tissues of volunteers and their $\mathrm{T} 1 \rho$ values $\left(\mathrm{Y}=-10.43 \mathrm{X}+75.60, \mathrm{R}^{2}=0.731\right)$ (Table 5, Figures 7,8).

To evaluate the fit degree of the $T 1 \rho$ values between the third experimental group model samples and the lumbar intervertebral disc nucleus pulposus tissues of volunteers, the in vitro model sample proteoglycan concentration of $8-10 \%$ was set as Pfirrmann III, $11-16 \%$ as Pfirrmann II, and $17-20 \%$ as Pfirrmann I. The regression equation between the Pfirrmann grading and T1 $\rho$ values of the in vitro model samples in the third experimental group was obtained $\left(\mathrm{Y}=-8.69 \mathrm{X}+133.6, \mathrm{R}^{2}=0.765\right)$ (Figure 8). The covariance comparison of the slope of the regression 
Table 4 T1rho (T1 $\rho)$ values of lumbar intervertebral disc nucleus pulposus in 4 volunteers with no low back pain (ms)

\begin{tabular}{lcccc}
\hline Lumbar disc & No. 1 T1 $\rho$ value $(\mathrm{ms})$ & No. 2 T1 $\rho$ value $(\mathrm{ms})$ & No. 3 T1 $\rho$ value $(\mathrm{ms})$ & No. $4 \mathrm{~T} 1 \rho$ value $(\mathrm{ms})$ \\
\hline Lumbar 1/2 & $123.8 \pm 0.5$ & $118.8 \pm 0.6$ & $124.7 \pm 1.0$ & $124.3 \pm 0.7$ \\
Lumbar 2/3 & $125.6 \pm 0.4$ & $110.3 \pm 0.6$ & $116.9 \pm 0.4$ & $117.8 \pm 0.5$ \\
Lumbar 3/4 & $119.9 \pm 0.5$ & $112.6 \pm 0.7$ & $112.3 \pm 0.5$ & $119.3 \pm 0.2$ \\
Lumbar 4/5 & $77.6 \pm 0.7$ & $82.7 \pm 0.2$ & $101.3 \pm 0.7$ & $86.3 \pm 0.6$ \\
Lumbar 5/sacral 1 & $85.8 \pm 0.7$ & $69.8 \pm 0.5$ & $97.6 \pm 0.5$ & $73.7 \pm 0.4$ \\
\hline
\end{tabular}

Table 5 Pfirrmann grading of lumbar intervertebral discs of 4 volunteers with no low back pain

\begin{tabular}{lcccc}
\hline Lumbar disc & No. 1 & No. 2 & No. 3 & No. 4 \\
\hline Lumbar 1/2 & I & II & I & I \\
Lumbar 2/3 & I & II & II & II \\
Lumbar 3/4 & II & III & II & II \\
Lumbar 4/5 & IV & IV & III & IV \\
Lumbar 5/sacral 1 & V & V & IV & V \\
\hline
\end{tabular}

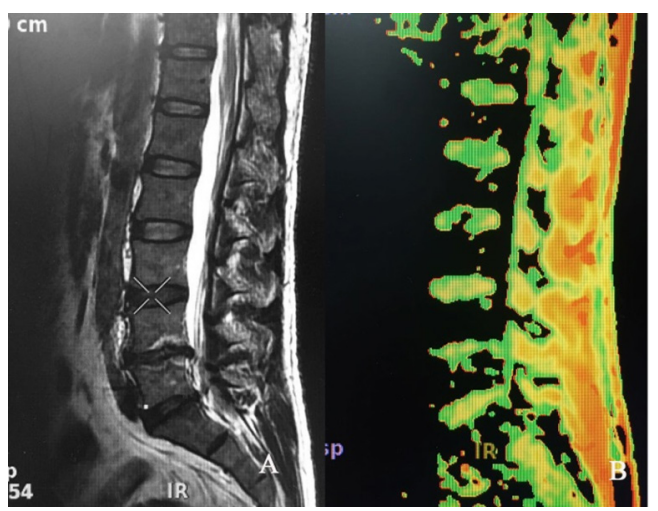

Figure 7 Sequence images. (A) Magnetic resonance T2 weighted image (MR-T2WI) sequence images of volunteers' lumbar intervertebral discs; (B) Magnetic resonance T1rho (MR-T1 $\rho$ ) sequence pseudo-color images.

equation between the Pfirrmann grading and T1 $\rho$ values between the in vitro model samples in the third experimental group and the lumbar intervertebral disc nucleus pulposus tissues of volunteers indicated no significant differences between them $(\mathrm{F}=0.54, \mathrm{P}=0.814)$, suggesting that the slope of regression equation between the Pfirrmann grading and T1 $\rho$ value in the in vitro model samples and lumbar disc nucleus tissues of volunteers was a good fit.

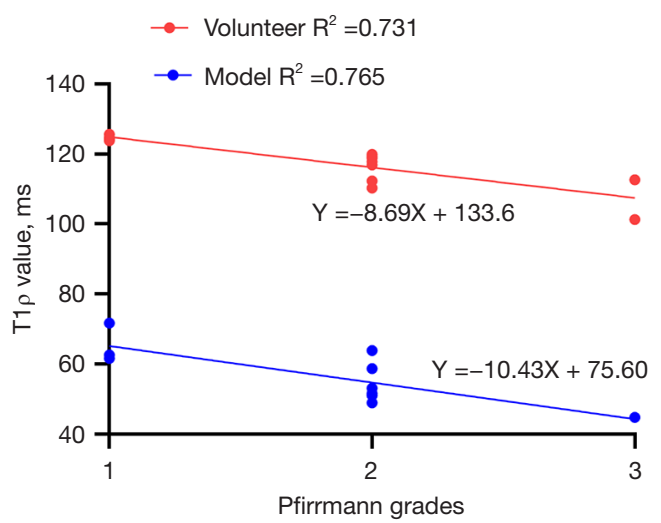

Figure 8 Scatterplot and regression line of the regression equation between the Pfirrmann grading and T1rho (T1 $\rho)$ value of the model samples in the third experimental group and volunteers.

\section{Discussion}

Approximately $80 \%$ of people worldwide will experience low back pain during their lifetime, and $5 \%$ will develop chronic low back pain, which affects quality of life and ability to work (11). Degeneration of the lumbar intervertebral disc is the main cause of low back pain. China has a large, aging population, so early diagnosis and the prevention and treatment of this disease it is of great economic.

The most accurate diagnostic method for early degeneration of lumbar intervertebral disc is to directly obtain the nucleus pulposus tissue to quantitatively measure the biochemical components, but this method is associated with invasive injury to the intervertebral disc, which will aggravate the degeneration of the intervertebral disc, rendering it unsuitable for clinical application (12). Studies have shown that the main biochemical change in the early degeneration of the intervertebral disc is the reduction of proteoglycan in the nucleus pulposus tissue (13). Therefore, there is a clinical need for a non-invasive, quantitative 
method to detect the proteoglycan content in the nucleus pulposus tissue of the intervertebral disc.

Lumbar X-rays and computed tomography scans cannot diagnose early disc degeneration, and conventional magnetic resonance T2WI sequencing cannot quantitatively evaluate the degree of lumbar disc degeneration. At present, only MR-T1 $\rho$ and T2-mapping imaging technology can be used to quantitatively measure early lumbar disc degeneration without invasive injury (12). Studies have shown that using MR-T1 $\rho$ sequence to quantitatively evaluate nucleus pulposus tissue proteoglycan is superior to other magnetic resonance sequences (14). Because MR-T1 $\rho$ imaging technology is sensitive to the low-frequency movement of macromolecular substances, and the proteoglycan molecules in the nucleus pulposus tissue of the intervertebral disc are macromolecular substances, MR-T1 $\rho$ technology can be used to quantitatively evaluate the proteoglycan content in the nucleus pulposus tissue of the intervertebral disc $(15,16)$. Previous studies have shown that the T1 $\rho$ relaxation time is significantly and positively correlated with the proteoglycan content of human or animal lumbar intervertebral discs $(17,18)$. Our research group has researched the natural degeneration of lumbar intervertebral discs in rhesus monkeys, and confirmed that the proteoglycan content of the lumbar disc nucleus pulposus of rhesus monkeys is positively correlated with the T1 $\rho$ value. However, we also found that the T1 $\rho$ value of the lumbar intervertebral disc from the same rhesus monkey was significantly different when scanned by different MRI equipment. Therefore, the T1 $\rho$ value cannot be directly used to accurately diagnose the early degeneration of the human lumbar intervertebral disc without calibration.

The quantification and calibration of $T 1 \rho$ values are technical difficulties in the clinical setting (19), and some methods have been reported to calibrate $\mathrm{T} 1 \rho$ values to increase the detection rate of diseases (20-22). In the present study, we set up in vitro model samples with different proteoglycan content or water content to simulate different degenerated nucleus pulposus tissues. In the in vitro model in the first experimental group, the results showed that more defective areas were seen in the MR-T1 $\rho$ image of the modeled samples. After changing the experimental conditions, some spots or flaky defect areas still could still be seen from the MR-T1 $\rho$ image of the in vitro model in the second experimental group. After another conditional improvement, the MR-T1 $\rho$ image showed no obvious defect area in the modeled samples in the third experimental group, and the proteoglycan concentration was positively correlated with the T1 $\rho$ value. The in vitro model samples in the third group underwent MR-T1 $\rho$ imaging re-scanning after being sterilized by ultraviolet rays and sealed at a low temperature $\left(10-15^{\circ} \mathrm{C}\right)$ for 3 months. According to the ICC value, the biochemical components of the model samples were still stable after a long period of storage. The results of these 3 groups confirmed that the ideal in vitro model with stable biochemical components needs a higher water content and the cryopreserved samples should be aseptically seal.

We selected 4 adult volunteers with no low back pain to perform the T1 $\rho$ sequence scan and T2WI sequence scan with the same parameters, and evaluated the Pfirrmann grading of their lumbar intervertebral discs. The results showed that there were no obvious differences between the slope of the regression equation between the Pfirrmann grading and T1 $\rho$ value of the in vitro model samples in the third experimental group and the lumbar intervertebral disc tissue of volunteers, suggesting that this model has a good degree of fit with the degeneration of the nucleus pulposus tissue of human lumbar intervertebral disc.

\section{Conclusions}

In this experiment, we successfully constructed an in vitro model of MR-T1 $\rho$ to quantitatively evaluate the proteoglycans of nucleus pulposus tissue. However, to accurately quantify the proteoglycan content of human lumbar intervertebral disc nucleus pulposus by clinical MRT1 $\rho$ imaging technology, large samples are required to establish an in vitro model database and post-processing platform to accurately detect theMR-T1 $\rho$ value of human lumbar intervertebral disc nucleus pulposus tissue, and to provide a scientific method for the accurate diagnosis of early degeneration of the lumbar intervertebral disc.

\section{Acknowledgments}

Funding: None.

\section{Footnote}

Reporting Checklist: The authors have completed the MDAR reporting checklist. Available at https://dx.doi. org/10.21037/atm-21-4297

Data Sharing Statement: Available at https://dx.doi. org/10.21037/atm-21-4297 
Conflicts of Interest: All authors have completed the ICMJE uniform disclosure form (available at https://dx.doi. org/10.21037/atm-21-4297). The authors have no conflicts of interest to declare.

Ethical Statement: The authors are accountable for all aspects of the work in ensuring that questions related to the accuracy or integrity of any part of the work are appropriately investigated and resolved.

Open Access Statement: This is an Open Access article distributed in accordance with the Creative Commons Attribution-NonCommercial-NoDerivs 4.0 International License (CC BY-NC-ND 4.0), which permits the noncommercial replication and distribution of the article with the strict proviso that no changes or edits are made and the original work is properly cited (including links to both the formal publication through the relevant DOI and the license). See: https://creativecommons.org/licenses/by-nc-nd/4.0/.

\section{References}

1. Benzakour T, Igoumenou V, Mavrogenis AF, et al. Current concepts for lumbar disc herniation. Int Orthop 2019;43:841-51.

2. Yaltirik CK, Timirci-Kahraman Ö, Gulec-Yilmaz S, et al. The Evaluation of Proteoglycan Levels and the Possible Role of ACAN Gene (c.6423T>C) Variant in Patients with Lumbar Disc Degeneration Disease. In Vivo 2019;33:413-7.

3. Zuo J, Joseph GB, Li X, et al. In vivo intervertebral disc characterization using magnetic resonance spectroscopy and T1 $\rho$ imaging: association with discography and Oswestry Disability Index and Short Form-36 Health Survey. Spine (Phila Pa 1976) 2012;37:214-21.

4. Zhang SF, Zhang B,Wang GH, et al. The application value of 3.0 T T1 $\rho$ and T2 mapping imaging in the study of cervical disc degeneration in patients. Chinese Journal of Radiology 2019;53:732-6.

5. Johannessen W, Auerbach JD, Wheaton AJ, et al. Assessment of human disc degeneration and proteoglycan content using T1rho-weighted magnetic resonance imaging. Spine (Phila Pa 1976) 2006;31:1253-7.

6. Pietrosimone B, Pfeiffer SJ, Harkey MS, et al. Quadriceps weakness associates with greater $\mathrm{T} 1 \rho$ relaxation time in the medial femoral articular cartilage 6 months following anterior cruciate ligament reconstruction. Knee Surg Sports Traumatol Arthrosc 2019;27:2632-42.
7. Van Rossom S, Smith CR, Zevenbergen L, et al. Knee Cartilage Thickness, T1 $\rho$ and T2 Relaxation Time Are Related to Articular Cartilage Loading in Healthy Adults. PLoS One 2017;12:e170002.

8. Hänninen NE, Nykänen O, Prakash M, et al. Orientation anisotropy of quantitative MRI parameters in degenerated human articular cartilage. J Orthop Res 2021;39:861-70.

9. Xiong X, Zhou Z, Figini M, et al. Multi-parameter evaluation of lumbar intervertebral disc degeneration using quantitative magnetic resonance imaging techniques. Am J Transl Res 2018;10:444-54.

10. Zhou Z, Jiang B, Zhou Z, et al. Intervertebral disk degeneration: T1 $\rho$ MR imaging of human and animal models. Radiology 2013;268:492-500.

11. Becker BA, Childress MA. Nonspecific Low Back Pain and Return To Work. Am Fam Physician 2019;100:697-703.

12. Melrose J, Ghosh P, Taylor TK. A comparative analysis of the differential spatial and temporal distributions of the large (aggrecan, versican) and small (decorin, biglycan, fibromodulin) proteoglycans of the intervertebral disc. J Anat 2001;198:3-15.

13. Sampara P, Banala RR, Vemuri SK, et al. Understanding the molecular biology of intervertebral disc degeneration and potential gene therapy strategies for regeneration: a review. Gene Ther 2018;25:67-82.

14. Duvvuri U, Goldberg AD, Kranz JK, et al. Water magnetic relaxation dispersion in biological systems: the contribution of proton exchange and implications for the noninvasive detection of cartilage degradation. Proc Natl Acad Sci U S A 2001;98:12479-84.

15. Belavý DL, Quittner MJ, Ridgers N, et al. Running exercise strengthens the intervertebral disc. Sci Rep 2017;7:45975.

16. Chen L, Liao J, Klineberg E, et al. Small leucine-rich proteoglycans (SLRPs): characteristics and function in the intervertebral disc. J Tissue Eng Regen Med 2017;11:602-8.

17. Peixoto-Santos JE, Kandratavicius L, Velasco TR, et al. Individual hippocampal subfield assessment indicates that matrix macromolecules and gliosis are key elements for the increased $\mathrm{T} 2$ relaxation time seen in temporal lobe epilepsy. Epilepsia 2017;58:149-59.

18. Ying J, Han Z, Zeng Y, et al. Evaluation of intervertebral disc regeneration with injection of mesenchymal stem cells encapsulated in PEGDA-microcryogel delivery system using quantitative T2 mapping: a study in canines. Am J Transl Res 2019;11:2028-41.

19. Chen W. Errors in quantitative T1rho imaging and 
Page 12 of 12

the correction methods. Quant Imaging Med Surg 2015;5:583-91.

20. Qi H, Bustin A, Kuestner T, et al. Respiratory motioncompensated high-resolution 3D whole-heart T1 $\rho$ mapping. J Cardiovasc Magn Reson 2020;22:12.

21. Andronesi OC, Nicholson K, Jafari-Khouzani K, et al. Imaging Neurochemistry and Brain Structure Tracks Clinical Decline and Mechanisms of ALS in Patients.

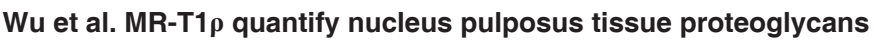

Front Neurol 2020;11:590573.

22. Gram M, Seethaler M, Gensler D, et al. Balanced spinlock preparation for B1 -insensitive and B0 -insensitive quantification of the rotating frame relaxation time T1 $\rho$. Magn Reson Med 2021;85:2771-80.

(English Language Editor: R. Scott)
Cite this article as: $\mathrm{Wu} \mathrm{Z,} \mathrm{Li} \mathrm{J,} \mathrm{Chen} \mathrm{L,} \mathrm{Chen} \mathrm{S,} \mathrm{Zhuang} \mathrm{W.}$ Establishing an in vitro model of MR-T1 $\rho$ imaging technology to quantify nucleus pulposus tissue proteoglycans: a preliminary study. Ann Transl Med 2021;9(20):1528. doi: 10.21037/atm-21-4297 